\title{
Aumento da expressão do MCP-1 coroidal e escleral em modelo experimental de hipercolesterolemia
}

\author{
Increase of choroidal and scleral MCP-1 expression in hypercholesterolemia experimental model \\ Rogil José de Almeida Torres ${ }^{1}$, Lucia de Noronha², Antonio Marcelo Barbante Casella ${ }^{3}$, Thaís Isabel Lumikoski ${ }^{4}$, Leonardo Brandão Précoma ${ }^{4}$, \\ Caroline luzia de Almeida Torres ${ }^{5}$, Andréa Luchini ${ }^{6}$, Mario Claudio Soares Sturzeneker ${ }^{7}$, Dalton Bertolim Précoma ${ }^{8}$
}

\section{RESUMO}

Objetivo: $O$ objetivo deste trabalho é demonstrar experimentalmente que a dieta rica em colesterol provoca aumento da expressão da MCP-1 na coroide e esclera.

Método: Coelhos New Zealand foram organizados em dois grupos: GN (grupo dieta normal), composto por 8 coelhos (8 olhos), recebeu ração padrão para coelhos, durante 4 semanas; GH (grupo hipercolesterolêmico), composto por 13 coelhos (13 olhos), recebeu dieta rica em colesterol a $1 \%$ por 8 semanas. Foi realizada a dosagem sérica de colesterol total, triglicerídeos, HDL colesterol, glicemia de jejum no início do experimento e no momento da eutanásia. Ao final da $8^{\underline{a}}$ semana para o GH e $4^{\underline{a}}$ semana para o GN foi realizada a eutanásia dos animais e os olhos foram submetidos à análise imuno-histoquímica com o anticorpo anti-MCP-1.

Resultados: A dieta provocou significativo aumento do colesterol total e triglicerídeos do $\mathrm{GH}$ em relação ao $\mathrm{GN}(p<0,001)$. Houve significativo aumento da expressão da MCP-1 na coroide e esclera dos animais do GH em relação ao GN $(p<0,001)$.

Conclusão: Este estudo demonstrou que a dieta hipercolesterolêmica em coelhos induz ao aumento da expressão do MCP-1 na coroide e esclera.

Descritores: Ativação de macrófagos; Degeneração macular; Aterosclerose;Colesterol; Coroide; Esclera; Proteínas quimioatraentes de monócitos; Animais; Coelhos

\begin{abstract}
Purpose: The aim of this study is to experientially demonstrate that a cholesterol-enriched diet induces an increase in the MCP-1 expression in the choroid and sclera.

Method: New Zealand rabbits were divided into two groups: GN (normal diet group) of 8 rabbits (8 eyes) was fed a standard rabbit diet for 4 weeks; GH (hypercholesterolemic group) of 13 rabbits (13 eyes) was fed a $1 \%$ cholesterol enriched diet for 8 weeks. Total serum cholesterol, triglyceride, HDL cholesterol and fasting blood glucose exams were performed at the start of the experiment and at the euthanasia time. After GH $8^{\text {th }}$ week and GN $4^{\text {th }}$ week animals were euthanized and their eyes underwent immunohistochemical analysis with the anti-MCP-1 antibody.
\end{abstract}

Results: The diet has induced a significant increase in GH total cholesterol and triglyceride levels when compared with NG $(p<0.001)$. There was a significant increase in the MCP-1 expression in GH choroid and sclera in relation to $G N(p<0.001)$.

Conclusion: This study has revealed that the hypercholesterolemic diet in rabbits induces an increase in the MCP-1 expression in the choroid and sclera.

Keywords: Macrophage activation; Macular degeneration; Atherosclerosis; Cholesterol; Choroid; sclera; Monocyte chemoattractant proteins; Animals; Rabbits

\section{INTRODUÇÃO}

Já foi demonstrado experimentalmente que a dieta rica em colesterol provoca aumento do número de macrófagos na esclera e coroide ${ }^{(1-3)}$. Estas células, consideradas fundamentais para a origem da placa aterosclerótica ${ }^{(4)}$, têm também sido relacionadas com a degeneração macular relacionada à idade $(\mathrm{DMRI})^{(5-7)}$. Adicionalmente, foi sugerido que a doença macular degenerativa e a aterosclerose compartilham mecanismos fisiopatogênicos similares ${ }^{(8,9)}$. Corroborando estas afirmações, vários relatos têm demonstrado que citocinas inflamatórias, fatores de crescimento e enzimas que estão envolvidos na formação da placa aterosclerótica, podem ser importantes para a evolução da DMRI(5,10-12).
Mecanismos pelos quais os macrófagos acumulam-se na esclera e coroide não foram ainda totalmente elucidados. Sabe-se que a dislipidemia ocasiona disfunção endotelial, permitindo a penetração passiva do colesterol LDL (LDL) para íntima vascular. Neste local, a LDL sofre ação de espécies reativas de oxigênio (ERO), tornando-se oxidada. O LDL oxidada estimula as células endoteliais do vaso ao aumento da expressão de moléculas de adesão vascular (P-Selectin, Molécula 1 de adesão intercelular, também chamada de ICAM-1, e Molécula 1 de adesão de células vasculares ou V(AM-1) que, junto com os ligantes da proteína de quimioatração de monócitos (MCP-1) e seus receptores CCR2, facilitam a ativação de monócitos e adesão ao endotélio ${ }^{(4,13,14)}$. Sendo assim, a constatação do aumento da ex-
Submitted for publication: October 3,2011

Accepted for publication: November 26, 2011

Study carried out at the Surgical Technique Laboratory at PUC-PR and the Study Center of the Angelina Caron Hospital.

Physician, Pontifícia Universidade Católica do Paraná - PUCPR - Curitiba (PR), Brazil. 2 Patologist, Pontifícia Universidade Católica do Paraná - PUCPR - Curitiba (PR), Brazil.

${ }^{3}$ Physician, Universidade Estadual de Londrina - UEL - Londrina (PR), Brazil.

${ }^{4}$ Medical student, Pontifícia Universidade Católica do Paraná - PUCPR - Curitiba (PR), Brazil.

5 Medical student, Universidade Positivo, Curitiba (PR), Brazil.

${ }^{6}$ Medical student, Universidade Positivo, Curitiba (PR), Brazil.
${ }^{5}$ Pho Oftalmológico de Curitiba, Curitiba (PR), Brazil

Physician, Departamento de Cardiologia, Santa Casa de Misericórdia de Ponta Grossa, Ponta Grossa (PR), Brazil.

Professor, Departamento de Cardiologia, Pontifícia Universidade Católica do Paraná - PUCPR - Curitiba (PR), Brazil.
Funding: No specific financial support was available for this study.

Disclosure of potential conflicts of interest: R.J.A.Torres, None; L.Noronha, None; A.M.B.Casella, None; T.I.Lumikoski, None; L.B.Précoma, None; C.L.A.Torres, None; A.Luchini, None; M.C.S. Sturzeneker, None; D.B.Précoma, None.

Correspondence address: Rogil José de Almeida Torres. Rua Emiliano Perneta 390 - Conj. 1407 Curitiba (PR) - 80010-050 - Brazil - E-mail: rjat@terra.com.br

Projeto número 240/080. CEUA - Pontifícia Universidade Católica do Paraná. Registro do parecer no CEUA: 307 Data do parecer: 19/08/2008 
pressão de moléculas de adesão e/ou MCP-1 na coroide e esclera pode explicar a presença de macrófagos nestas estruturas, em modelo experimental de hipercolesterolemia.

O objetivo deste trabalho é demonstrar experimentalmente que a dislipidemia provoca aumento da expressão da MCP-1 na coroide e esclera.

\section{MÉTODOS}

Para a realização deste estudo, o protocolo foi aprovado pela Comissão de Ética em Experimentação Animal da Pontifícia Universidade Católica do Paraná, seguindo os princípios da Association for Research in Vision and Ophthalmology (ARVO).

\section{Ambiente de EXPERIMENTAÇÃo}

Os procedimentos deste estudo foram realizados nas dependências do laboratório de Técnica Operatória da PUC-PR e do Centro de Estudos do Hospital Angelina Caron (HAC). Os animais foram mantidos no biotério em macroambiente com ciclos de iluminação $12 / 12$ horas, com trocas de ar e temperatura controlada de 19 a $23^{\circ} \mathrm{C}$ e receberam durante o experimento água e ração específica para a espécie Nuvital ${ }^{\circledR}$ (Nuvital, Colombo, Brasil) de forma ad libitum.

\section{ANimais Utilizados E DELINEAMENTO EXPERIMENTAL}

Foram utilizados 21 coelhos machos albinos (Oryctolagus cunicullus), da linhagem New Zealand, procedentes do Biotério Central da Pontifícia Universidade Católica do Paraná, com idade média aproximada de 110 dias e peso médio de 2.770 gramas. Os animais foram divididos em dois grupos: grupo 1 (GN), composto por 8 coelhos, e grupo $2(\mathrm{GH})$ composto por 13 coelhos. O GN, ou grupo de deita normal, recebeu ração padrão para coelhos de laboratório Nuvital ${ }^{\circledR}$ (Nuvital, Colombo, Brasil) e foi submetido à eutanásia em 4 semanas. O GH, ou grupo hipercolesterolêmico, recebeu ração padrão para coelhos de laboratório Nuvital ${ }^{\circledR}$ (Nuvital, Colombo, Brasil), acrescida de colesterol a 1\%, em todo o período do estudo (ração suplementar). Este grupo foi submetido à eutanásia ao final de 8 semanas.

Cada coelho foi submetido à dosagem sérica de colesterol total, triglicerídeos, HDL colesterol, glicemia de jejum no início do experimento e no momento da eutanásia. As coletas das amostras sanguíneas foram realizadas através de punção da veia marginal auricular magna sob anestesia geral com injeção intramuscular de ketamina 5 mg/kg e xylazina 35 mg/kg. As dosagens plasmáticas de glicemia, colesterol total, colesterol HDL e triglicerídeos foram realizados pelo método enzimático colorimétrico automatizado (Architec ${ }^{\circledR}$ - Abbott). Uma soroteca com 500 microlitros de cada animal foi congelada para análises futuras. O peso dos coelhos foi aferido em rotina semanal.

Os animais foram sacrificados com injeção endovenosa de $5 \mathrm{ml}$ de pentobarbital e os olhos imediatamente fixados em paraformaldeído a 4\% (Merck, Darmstadt, Germany), em 0,1 M fosfato/pH 7,4 por 4 horas, para análise imuno-histoquímica.

\section{Preparo da ração suplementar}

A ração hipercolesterolêmica a 1\%, utilizada nas oito semanas do experimento, apresentava 200 gramas de colesterol (Sigma-Aldrich a $95 \%{ }^{\circledR}$ ) dissolvido em 800 mililitros de clorofórmio (Biotec ${ }^{\circledR}$ ), distribuídos homogeneamente em 20 quilogramas de ração Nuvital ${ }^{\circledR}$ (Nuvital, Colombo, Brasil). Antes de administrar a ração aos coelhos, esperou-se um período mínimo de 24 horas para adequada evaporação do clorofórmio. A quantidade diária ofertada para cada animal foi de 600 gramas ao dia ${ }^{(15)}$.

\section{Preparação do tecido e anÁlise imuno-histoquímica}

Os dois olhos de cada animal (total de 42) foram removidos e submetidos à fixação, porém somente um foi escolhido para o estudo, de forma aleatória. Depois da fixação os espécimes foram avaliados macroscopicamente, sendo feito uma secção coronal ao nível do nervo óptico, dividindo os globos oculares em duas metades iguais (superior e inferior). A metade inferior foi estocada para estudos posteriores. Já a metade superior foi submetida à desidratação, diafanização e impregnação em parafina, com histotécnico da marca Leica ${ }^{\circledR}$ (Leica, Wetzlar, Germany), modelo TP 1020. Para a confecção dos blocos de parafina utilizou-se o inclusor Leica ${ }^{\circledR}$, modelo EG1160. Estes blocos foram cortados com micrótomo, marca Leica ${ }^{\circledR}$ modelo RM2145 (Leica, Wetzlar, Germany), a 5 para obtenção dos cortes histológicos (total de 42). Estes cortes foram pescados em lâmina de vidro com albumina, corados com hematoxilina-eosina e montados com lamínula de vidro de 24×90 mm Entellan, Merck ${ }^{\circledR}$ (Merck, Darmstadt, Germany).

Os cortes histológicos foram desparafinizados e re-hidratados, sendo feito posteriormente o bloqueio da peroxidase endógena. Posteriormente foram lavados em água deionizada sendo incubados em câmara úmida a $95^{\circ} \mathrm{C}$ por 20 minutos para recuperação antigênica. Após esta fase, foi realizado novo bloqueio da peroxidase endógena. Os cortes foram cobertos com anticorpo primário policlonal produzido em camundongo denominado anti-MCP-1, da marca Imuny ${ }^{\circledR}$ (Imuny Biotechnology, Campinas, Brasil). Posteriormente, foi recoberto com anticorpo secundário, polímero marcado-HRP anticamundongo advance ${ }^{\circledR}$ System (DakoCytomation, Inc., Carpinteria, CA) e incubados, à temperatura ambiente, por 30 minutos. A seguir foram submetidos ao gotejamento de substrato misto recém preparado DAB (DakoCytomation, Inc., CA, USA). Novamente foram incubados por 3 até 5 minutos. Os cortes foram contracorados com hematoxilina de Mayer e depois montados.

Controles positivos e negativos foram usados em todas as marcações e as lâminas foram primeiramente analisadas por um observador, sem conhecimento prévio do grupo de identificação (de maneira cega). Nesta análise, foi anotada a presença ou não de positividade para o marcador anti-MCP-1. As áreas positivas adquiriram coloração acastanhada e foram analisadas pelo método da morfometria de cores. Para tanto, foram capturadas imagens de 5 campos consecutivos, de pars plana a pars plana contralateral, em objetiva de 40x, utilizando a câmera Olympus BX50, modelo DXC-107A e o software Image Pró Plus. Tal software permitiu que as áreas positivas fossem selecionadas e coloridas por um observador. A área imunorreativa foi automaticamente calculada pelo software. Estes dados foram enviados ao programa Microsoft Excel (Redmond, WA) em forma de planilhas para análise estatística. A variável área imunorreativa refere-se à somatória de todas as áreas positivas em cada um dos 5 campos analisados.

\section{AnÁlise estatística}

Para a comparação dos grupos definidos pelo tratamento em relação às variáveis quantitativas, foi considerado o teste t de Student para amostras independentes. Para a comparação entre as avaliações início e eutanásia dentro de cada grupo, foi usado o teste t de Student para amostras pareadas. A condição de normalidade foi avaliada pelo teste de Shapiro-Wilks. Variáveis que não apresentaram a condição de simetria foram submetidas a uma transformação logarítmica. Valores de $p<0,05$ indicaram significância estatística. Os dados foram analisados com o programa computacional Statistica v.8.0.

\section{RESULTADOS}

\section{COMPARAÇÃo dOS GRUPOS EM RELAÇÃo Às VARIÁVEIS: GLICOSE, COLESTEROL TOTAL, HDL E TRIGLICERÍDEOS}

O grupo dieta normal (GN) manteve as variáveis laboratoriais estáveis e normais do início ao fim do experimento. Por outro lado, os animais do GH (grupo dieta hipercolesterolêmica) manifestaram expressivo aumento do colesterol total na eutanásia. No momento 
basal, a média do colesterol total do GH era de $41,3 \mathrm{mg} / \mathrm{dl}$ e ao final observou-se significativa elevação para $2146,8 \mathrm{mg} / \mathrm{dl}(\mathrm{p}<0,001)$. Outra variável laboratorial que demonstrou importante variação no GH foram os triglicerídeos. No momento basal, a média dos triglicerídeos deste grupo foi de $46,5 \mathrm{mg} / \mathrm{dl}$, enquanto que na eutanásia chegou a 168,5 mg/dl $(p=0,001)$. A glicemia e o HDL colesterol não manifestaram variações significativas ao final do experimento neste grupo.

\section{ComparaÇão dos GRUPOS EM RELAÇÃo À ÁREA tOTAL IMUNORREATIVA AO MCP-1}

Houve aumento significativo da expressão do MCP-1 na coroide e esclera dos animais do GH (Tabela 1). A figura 1 demonstra o significativo aumento da espessura da coroide e esclera do GH (B) em relação ao GN (A). É possível verificar também que a imunorreatividade coroidal e escleral ao MCP-1 do GH foi superior ao GN. As áreas imunorreativas adquiriram coloração acastanhada.

\section{DISCUSSÃO}

Já foi demonstrado que a dislipidemia provoca disfunção do endotélio vascular e consequente penetração passiva da LDL na íntima do vaso. A LDL sofre processo de oxidação, estimulando as células endoteliais ao aumento da expressão de moléculas de adesão vascular (P-Selectin, ICAM e VCAM), da proteína de quimioatração de monócitos (MCP-1) e de seus receptores nos monócitos (CCR2). Esta sequência de eventos facilita a ativação dos monócitos e adesão destes ao endotélio ${ }^{(4,13,14)}$
No presente estudo, foi administrada dieta rica em colesterol para analisar a expressão da MCP-1 nos vasos da coroide. Como esperado, a dieta hipercolesterolêmica provocou significativo aumento do colesterol total e triglicerídeos do $\mathrm{GH}$ em relação ao $\mathrm{GN}(p \leq 0,001)$. É importante ressaltar que, o tempo de dieta rica em colesterol (8 semanas), assim como a concentração de colesterol (1\%), dada aos animais do $\mathrm{GH}$ foi maior que em outro estudo realizado pelo nosso grupo ${ }^{(3)}$. Este fato pode explicar a significativa diferença entre a espessura do complexo esclerocoroidal do GH em relação ao GN (Figura 1). Assim como ocorre nos grandes vasos ${ }^{(4,13,14)}$, a dislipidemia provocou significativo aumento da expressão da MCP-1 na coroide e esclera dos animais do GH (Tabela 1). MCP-1 é uma citocina quimiotática de baixo peso molecular (8-10 kDa) que está envolvida no recrutamento de monócitos para o local de inflamação ${ }^{(16-18)}$. Embora a MCP-1 não se expresse em vasos normais, vários estímulos, com destaque para a resposta à oxidação de lipídeos, podem induzir a liberação de citocinas inflamatórias. Estas citocinas promovem secreção de MCP-1 pelas células endoteliais, células musculares lisas vasculares e células do epitélio pigmentário da retina (EPR) que conduz ao acúmulo de leucócitos e perpetua a reação inflamatória ${ }^{(17-21)}$.

$\mathrm{Na}$ aterosclerose, está muito bem definida a participação desta citocina quimiotática na formação da placa ateromatosa. A MCP-1 atrai os monócitos da circulação para o endotélio vascular. Estas células diferenciam-se em macrófagos que, através de "scavenger receptors" (CD36 e SR-A), absorvem a LDL oxidada e formam os histiócitos. Os histiócitos expressam citocinas inflamatórias, enzimas e fatores de crescimento que, ao lado das citocinas liberadas pelas células T ativadas, promovem processo inflamatório, proliferação e migração de

Tabela 1. Área esclerocoroideana imunorreativa ao MCP-1 entre os grupos GH e GN

\begin{tabular}{lcccccccc}
\hline Variável & Grupo & $\mathbf{n}$ & Média & Mediana & Mínimo & Máximo & Desvio padrão & Valor de $\mathbf{p}^{*}$ \\
\hline Área imunorreativa & $\mathrm{GH}$ & 13 & 4346,8 & 3768,8 & 1213,1 & 8735,8 & 2427,1 & \\
& $\mathrm{GN}$ & 8 & 1483,0 & 1425,4 & 299,3 & 2713,8 & 797,3 & 0,001 \\
\hline
\end{tabular}

$\mathrm{GH}=$ grupo dieta rica em colesterol; $\mathrm{GN}=$ grupo dieta normal

* $=$ teste $t$ de Student para amostras independentes, $p<0,05$

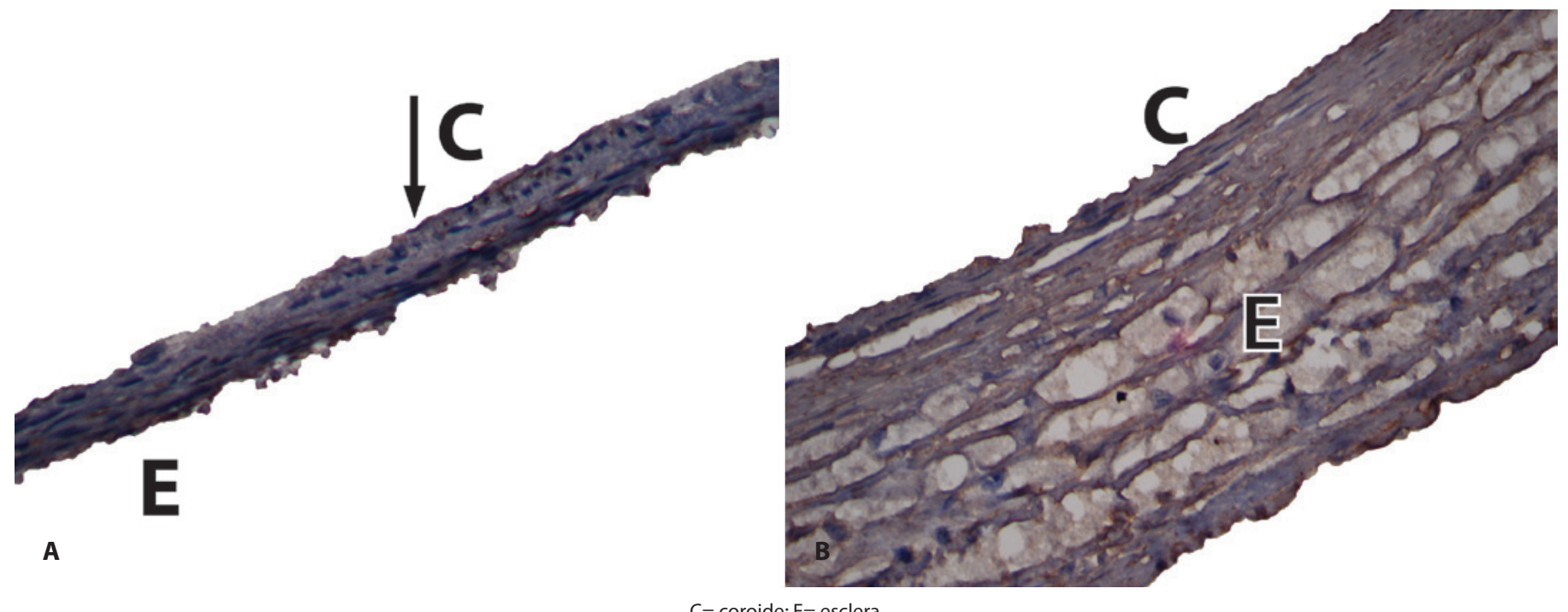

$\mathrm{C}=$ coroide; $\mathrm{E}=$ esclera

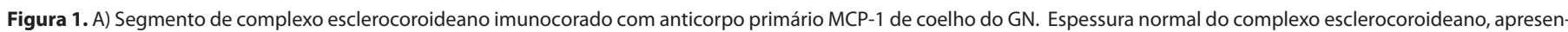

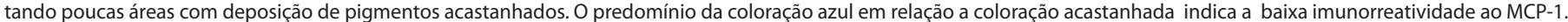

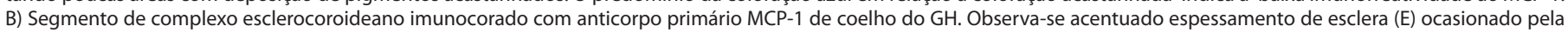

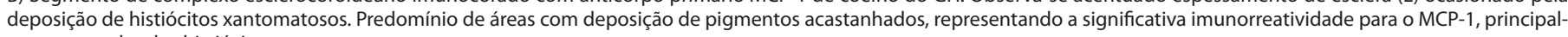
mente ao redor dos histiócitos xantomatosos. 
células musculares lisas e células endoteliais para dentro da íntima do vaso, formando assim a placa aterosclerótica ${ }^{(4,13,14)}$. Considerando que a hipercolesterolemia é um dos fatores modificáveis da degeneração macular relacionada à idade (DMRI)(22), e que citocinas, enzimas e fatores de crescimento responsáveis pela formação da placa ateromatosa também podem ter participação na gênese da doença macular degenerativa ${ }^{(5,10-12)}$, espera-se que a MCP-1 desempenhe igual função nos vasos da coroide.

Ao induzirmos a hipercolesterolemia, tentamos criar neste experimento condição fisiopatogênica similar à DMRI. Ou seja, acúmulo de lipídeos sub-retiniano que atrai macrófagos com objetivo de absorver a LDL oxidada. É importante lembrar que, independentemente do aumento do colesterol sérico, o EPR é fonte contínua de produção de lipídeos e ésteres de colesterol(23). Não menos importante é a constatação de que lipídios oxidados são encontrados na fase inicial da DMRI ${ }^{(23)}$. Estes depósitos originam-se da falha na degradação final de produtos resultantes da fagocitose dos segmentos externos das células fotorreceptoras, ricas em ácidos graxos poli-insaturados e vitamina $\mathrm{A}^{(24)}$. Os lipídeos oxidados estimulam a expressão de fatores quimiotáticos, tais como IL-8 e MCP-1, pelas células do EPR, os quais têm potencial para recrutar macrófagos ${ }^{(25)}$. Recente estudo demonstrou que a MCP-1 contribui para apoptose das células do epitélio pigmentário da retina, podendo assim desencadear a DMRI (26). Sendo assim, o modelo adotado neste estudo também induz à expressão de MCP-1 na coroide e esclera. Isto explica a presença de macrófagos no espaço sub-retiniano e consequente aumento de espessura do complexo esclerocoroideano observada neste e em outros relatos ${ }^{(1-3)}$.

Por outro lado, a relação entre o aumento da expressão da MCP-1, e consequente presença de macrófagos, ao desenvolvimento da DMRI deva ser feita cautelosamente. Já foi demonstrado que o recrutamento de macrófagos para dentro do espaço sub-retiniano ocorre de forma normal e fisiológica em olhos humanos, com a finalidade de remover seus debris ${ }^{(27)}$. Além do que, o único modelo experimental que simulou até o momento a DMRI humana, induzindo a formação de drusas, deposição de lipofuscina no EPR e neovascularização coroideana, foi realizado em camundongos geneticamente deficientes para C Cl-2 (MCP-1) e seu cognato no monócito CCCR-2 ${ }^{(28)}$. Neste estudo, os animais com deficiência de MCP-1 e Ccr-2 tiveram o recrutamento de macrófagos prejudicado para o espaço sub-retiniano. Devido a esta disfunção, houve acúmulo de debris, de complementos (C5), e imunoglobulinas (lgG). Estas alterações possivelmente induziram à formação de drusas, deposição de lipofuscina no EPR e liberação de fator de crescimento do endotélio vascular (VEGF) pelas células do EPR, resultando no desenvolvimento de membrana neovascular sub-retiniana (MNSR) ${ }^{(28)}$.

Ao constatar que a DMRI e a aterosclerose apresentam mecanismos fisiopatogênicos similares ${ }^{(4-9)}$, espera-se que os medicamentos utilizados para o tratamento destas doenças tenham características e desempenhos equivalentes. Experimentalmente, já foi demonstrado que a ativação do receptor gama ativado pelo proliferador de peroxissomo (PPAR gama), utilizado com algum êxito na doença aterosclerótica, foi efetiva em reduzir o número de macrófagos no complexo esclerocoroideano e inibir a formação de membrana neovascular sub-retiniana ${ }^{(29,30)}$. Sabe-se que esta modalidade de medicamento reduz a expressão de citocinas inflamatórias, enzimas e fatores de crescimento, inibindo consequentemente o recrutamento de macrófagos ${ }^{(29,30)}$. Desta forma, fica evidente que o entendimento da arquitetura inflamatória na DMRI e seus fatores de risco mostram-se cada vez mais importantes no cenário atual.

Sendo assim, uma abordagem terapêutica da DMRI que vise interferir na ação dos macrófagos deverá ser muito bem ponderada. Talvez, na fase inicial da DMRI, a neutralização dos macrófagos possa piorar o curso da doença, visto que haverá maior acúmulo de debris, imunoglobulinas e complemento(28). Na fase tardia da DMRI, a supres- são dos macrófagos poderá ser interessante por neutralizar citocinas inflamatórias, como interleucinas e TNF-alfa, enzimas, como a MMP-9 que degrada a membrana basal, e fatores de crescimento, como o VEGF, que promove a angiogênese $\mathrm{e}^{(29,30)}$.

\section{CONCLUSÃO}

Este trabalho demonstrou que o aumento da expressão da MCP-1, no modelo experimental de hipercolesterolemia, pode explicar o acúmulo de macrófagos na coroide e esclera. Futuras pesquisas, utilizando drogas que interfiram nesta citocina quimiotática poderão esclarecer eventual utilidade no tratamento da DMRI.

\section{REFERÊNCIAS}

1. Queiroz JM, Viana, JC, Silva FG. Lesões oculares na hipercolesterolemia experimental do coelho em relação à patogênese da retinite de Coats. Rev Assoc Med Brasil. 1958:4:328-38.

2. Salazar JJ, Ramírez Al, de Hoz R, Rojas B, Ruiz E, Tejerina T, et al. Alterations in the choroid in hypercholesterolemic rabbits: reversibility after normalization of cholesterol levels. Exp Eye Res. 2007:84(3):412-22.

3. Torres RJ, Maia M, Noronha L, Farah ME, Luchini A, Brik D, et al. [Evaluation of choroid and sclera early alterations in hypercholesterolemic rabbits: histologic and histomorphometric study]. Arq Bras Oftalmol. 2009:72(1):68-74. Portuguese.

4. Libby P. Inflammation in atherosclerosis. Nature. 2002;420(6917):868-74.

5. Oh H, Takagi H, Takagi C, Suzuma K, Otani A, Ishida K, et al. The potencial angiogenic role of macrophages in the formation of choroidal neovascular membranes. Invest Ophthalmol Vis Sci. 1999;40(9):1891-8.

6. Sakurai E, Anand A, Ambati BK, van Rooijen N, Ambati J. Macrophage depletion inhibits experimental choroidal neovascularization. Invest Ophthalmol Vis Sci. 2003;44(8): 3578-85

7. Cousins SW, Espinosa-Heidmann DG, Csaky KG. Monocyte activation in patients with age-related macular degeneration. Arch Ophthalmol. 2004:122(7):1013-8.

8. Friedman E. A hemodynamic model of the pathogenesis of age-related macular degeneration. Am J Ophthalmol. 1997;124(5):677-82. Comment in: Am J Ophthalmol. 2000; 130(5):658-63.

9. Friedman $\mathrm{E}$. The role of the atherosclerotic process in the pathogenesis of age-related macular degeneration. Am J Ophthalmol. 2000;130(5):658-63. Comment on: Am J Ophthalmol. 1997;124(5):677-82.

10. Steen B, Sejersen S, Berglin L, Seregard S, Kvanta A. Matrix metalloproteinases and metalloproteinases inhibitors in choroidal neovascular membranes. Invest Ophthalmol Vis Sci. 1998;39(11):2194-200.

11. Grossniklaus HE, Ling JX, Wallace TM, Dithmar S, Lawson DH, Cohen C, et al. Macrophage and retinal pigment epithelium expression of angiogenic cytokines in choroidal neovascularization. Mol Vis. 2002;8:119-26.

12. Sakurai E, Taguchi H, Anand A, Ambati BK, Gragoudas ES, Miller JW,et al.. Targeted disruption of the CD18 or ICAM-1 gene inhibits choroidal neovascularization. Invest Ophthalmol Vis Sci. 2003;44(6):2743-9.

13. Ross R. Atherosclerosis: an inflammatory disease. N Engl J Med. 1999;340(2):115-26. Comment in: N Engl J Med.1999;340(24):1928-9. N Engl J Med. 1999;340(24):1928; author reply 1929.

14. Nicolletti A, Caligiuri G, Hansson GK. Immunomodulation of atherosclerosis: myth and reality. J Intern Med. 2000;247(3):397-405

15. Sun YP, Lu NC, Parmley YWW, Hollenbeck CB. Effects of cholesterol diets on vascular function and atherogenesis in rabbits. Proc Soc Exp Biol Med. 2000;224(3):166-71.

16. Sallusto F, Mackay CR, Lanzavecchia A. The role of chemokine receptors in primary, effector, and memory immune responses. Annu Rev Immunol. 2000;18:593-620.

17. Charo IF, Taubman MB. Chemokines in the pathogenesis of vascular disease. Circ Res. 2004;95(9):858-66.

18. Weber C, Schober A, Zernecke A. Chemokines: key regulators of mononuclear cell recruitment in atherosclerotic vascular disease. Arterioscler Thromb Vasc Biol. 2004; 24(11):1997-2008

19. Nelken NA, Coughlin SR, Gordon D, Wilcox JN. Monocyte chemoattractant protein-1 in human atheromatous plaques. J Clin Invest. 1991;88(4):1121-7.

20. Yla-Herttuala S, Lipton BA, Rosenfeld ME, Sarkioja T, Yoshimura T, Leonard EJ, et al. Expression of monocyte chemoattractant protein 1 in macrophage-rich areas of human and rabbit atherosclerotic lesions. Proc Natl Acad Sci U S A. 1991;88(12):5252-6.

21. Holtkamp GM, DeVos AF, Peek R, Kijlsta A. Analysis of the secretion pattern of monocyte chemotactic protein-1 (MCP-1) and transforming growth factor-beta 2 (TGF-beta2) by human retinal pigment epithelial cells. Clin Exp Immunol. 1999;118(1):35-40.

22. Torres RJ, Maia M, Muccioli C, Winter G, Souza GK, Pasqualotto LR, et al. [Modifiable risk factors for age-related macular degeneration]. Arq Bras Oftalmol. 2009;72(3): 406-12. Portuguese.

23. Ruberti JW, Curcio CA, Millican CL, Menco BP, Huang JD, Johnson M. Quick-freeze/ deep-etch visualization of age-related lipid accumulation in Bruch's membrane. Invest Ophthalmol Vis Sci. 2003;44(4):1753-9.

24. Mata NL, Weng J, Travis GH. Biosynthesis of a major lipofuscin fluorophore in mice 
and humans with ABCR-mediated retinal and macular degeneration. Proc Natl Acad Sci U.S.A. 2000;9:7154-9

25. Higgins GT, Wang JH, Dockery P, Cleary PE, Redmond HP. Induction of angiogenic cytokine expression in cultured RPE by ingestion of oxidized photoreceptor outer segments. Invest Ophthalmol Vis Sci. 2003;44:1775-82.

26. Yang D, Elner SG, Chen X, Field MG, Petty HR, Elner VM. MCP-1-activated monocytes induce apoptosis in human retinal pigment epithelium. Invest Ophthalmol Vis Sci. 2011;52(8):6026-34

27. Nicolai $U$, Eckardt $C$. The occurrence of macrophages in the retina and periretinal tissues in ocular diseases. Ger J Ophthalmol. 1993;2:195-201
28. Ambati J, Anand A, Fernandez S, Sakurai E, Lynn BC, Kuziel WA, Rollins BJ, Ambati BK. An animal model of age-related macular degeneration in senescent CCl-2- or Ccr-2deficient mice. Nat Med. 2003;9(11):1390-7.

29. Torres RJ, Muccioli C, Maia M, Noronha L, Luchini A, Alessi A, Olandoski M, Farah ME, Précoma DB. Sclerochorioretinal abnormalities in hypercholesterolemic rabbits treated with rosiglitazone. Ophthalmic Surg Lasers Imaging. 2010;41(5): 562-71.

30. Murata T, He S, Hangai M, Ishibashi T, Xi XP, Kim S, Hsueh WA, Ryan SJ, Law RE, Hinton DR. Peroxisome proliferator-activated receptor-gamma ligands inhibit choroidal neovascularization. Invest Ophthalmol Vis Sci. 2000;41:2309-17. 